\title{
Magnetic topology of Co-based inverse opal-like structures
}

\author{
N. A. Grigoryeva, ${ }^{1}$ A. A. Mistonov, ${ }^{1}$ K. S. Napolskii, ${ }^{2}$ N. A. Sapoletova, ${ }^{2}$ A. A. Eliseev, ${ }^{2}$ W. Bouwman, ${ }^{3}$ D. V. Byelov, \\ A. V. Petukhov, ${ }^{4}$ D. Yu. Chernyshov,${ }^{5}$ H. Eckerlebe, ${ }^{6}$ A. V. Vasilieva, ${ }^{7}$ and S. V. Grigoriev ${ }^{7}$ \\ ${ }^{1}$ Faculty of Physics, Saint-Petersburg State University, 198504 Saint Petersburg, Russia \\ ${ }^{2}$ Department of Materials Science, M. V. Lomonosov Moscow State University, 119991 Moscow, Russia \\ ${ }^{3}$ Faculty of Applied Sciences, Delft University of Technology, Mekelweg 15, NL-2629 JB Delft, The Netherlands \\ ${ }^{4}$ van $t$ Hoff laboratory, Debye Institute for Nanomaterials Science, Utrecht University, NL-3584 CH Utrecht, The Netherlands \\ ${ }^{5}$ Swiss-Norwegian Beamlines at the European Synchrotron Radiation Facility, F-38000 Grenoble, France \\ ${ }^{6}$ GKSS Forschungszentrum, D-21502 Geesthacht, Germany \\ ${ }^{7}$ Petersburg Nuclear Physics Institute, Gatchina, 188300 Saint Petersburg, Russia
}

(Received 22 December 2010; revised manuscript received 30 May 2011; published 11 August 2011)

\begin{abstract}
The magnetic and structural properties of a cobalt inverse opal-like crystal have been studied by a combination of complementary techniques ranging from polarized neutron scattering and superconducting quantum interference device (SQUID) magnetometry to $\mathrm{x}$-ray diffraction. Microradian small-angle $\mathrm{x}$-ray diffraction shows that the inverse opal-like structure (OLS) synthesized by the electrochemical method fully duplicates the threedimensional net of voids of the template artificial opal. The inverse OLS has a face-centered cubic (fcc) structure with a lattice constant of $640 \pm 10 \mathrm{~nm}$ and with a clear tendency to a random hexagonal close-packed structure along the [111] axes. Wide-angle x-ray powder diffraction shows that the atomic cobalt structure is described by coexistence of $95 \%$ hexagonal close-packed and 5\% fcc phases. The SQUID measurements demonstrate that the inverse OLS film possesses easy-plane magnetization geometry with a coercive field of $14.0 \pm 0.5 \mathrm{mT}$ at room temperature. The detailed picture of the transformation of the magnetic structure under an in-plane applied field was detected with the help of small-angle diffraction of polarized neutrons. In the demagnetized state the magnetic system consists of randomly oriented magnetic domains. A complex magnetic structure appears upon application of the magnetic field, with nonhomogeneous distribution of magnetization density within the unit element of the OLS. This distribution is determined by the combined effect of the easy-plane geometry of the film and the crystallographic geometry of the opal-like structure with respect to the applied field direction.
\end{abstract}

DOI: 10.1103/PhysRevB.84.064405

PACS number(s): 61.05.fg, 81.07.-b, 42.70.Qs, 61.05.cc

\section{INTRODUCTION}

Photonic crystals based on artificial opals, the dielectric three-dimensional large-scale periodic structures formed by spherical submicrometer-sized colloidal particles, have attracted much attention in the past decade for their ability in light manipulation and for their promising potential applications in the area of photonics. Highly ordered artificial opals can be fabricated using high-quality monodisperse spheres made of silica or polystyrene using a combination of self-assembly and the sol-gel method. ${ }^{1}$ However, due to the relatively low dielectric contrast, the opals themselves cannot be used as good candidates for photonic applications. To enhance their interaction with light, the voids of a colloidal crystal are filled with other materialspolymers, ${ }^{2-4}$ liquids, ${ }^{5-7}$ inorganic oxides, ${ }^{8-11}$ carbon, ${ }^{12}$ semiconductors, ${ }^{13-16}$ superconductors, ${ }^{17-19}$ and metals. ${ }^{20-26}$ Then the original template can be removed, if necessary. This generates inverse opal-like structures (OLSs) whose size and chemical composition are tunable by varying the size of the colloids and the infiltration materials, respectively.

The applications of inverse opal structures are much wider than those involving only confinement and control of light (optical sensors, circuits, waveguides, etc.). OLSs can be additionally used as eliminators of unwanted heat from thermal emission sources, ${ }^{27}$ in the conventional lighting field for increasing efficiency and obtaining improved thermophotovoltaic devices, ${ }^{28}$ piezoelectric transducers, solar cells, phosphors, short-wavelength light emitters, ${ }^{29}$ gas sensors, ${ }^{30}$ and so on. The general interest in the study of nanoscale magnetic structures based on OLSs is related to their wide application in spintronic devices, hard disks, and high-density storage devices. Therefore, in order to design magnetic materials for specific applications, it is important to understand how macroscopic properties arise from the interplay of microscopic parameters such as nanoparticle size, interparticle exchange coupling, nanoparticle shape anisotropy, and structural anisotropy of the original template.

It is well known that the magnetic properties of nanostructured materials depend strongly on the size of the particles. As was shown in Ref. 31, the coercivity increases upon decrease of particle size; it is maximal at a size of typically some tens of nanometers and decreases drastically for smaller sizes. The magnetic properties are determined not solely by particle size but also by the exchange interactions between adjacent particles. Wernsdorfer and coauthors have investigated nanostructured magnets consisting of densely packed grains. ${ }^{32}$ They concluded that such nanomagnets are often magnetically soft as a result of the interactions between grains, in contrast to the isolated nanoparticles, which are magnetically hard with a large coercive field.

The magnetic correlations in nanostructured $\mathrm{Fe}, \mathrm{Co}$, and $\mathrm{Ni}$ samples made from consolidated nanoparticles have also been studied by small-angle neutron scattering. ${ }^{33,34}$ It was shown that the smallest correlation lengths of the order of the grain size were found in Co particles, down to around $20 \mathrm{~nm}$. The magnetic correlations are extended over many grains in $\mathrm{Fe}$, 
where they exceed $70 \mathrm{~nm}$, and for nanostructured $\mathrm{Ni}$, where the correlation length was equal to $150 \mathrm{~nm}$. The authors concluded that the magnetic moments in domains extending over several grains are tilted out of the external magnetic field direction in intermediate fields and the magnetization process does not proceed via domain-wall motion.

The investigations described in Refs. 32-34 were performed with spatially disordered systems. One can expect some similarity in the magnetic properties between spatially ordered and disordered nanosystems. Still, the structure can impose spatial periodicity and a preferred magnetization direction along certain crystallographic directions. In this paper the investigation of the magnetic properties of cobalt inverse opal-like structures is presented. One can consider an inverse OLS as an assembly of small metallic particles duplicating the shape of the voids and connected to each other via thin (several tens of nanometers) and long (several hundreds of nanometers) crosspieces. These voids are created by polystyrene spheres typically packed in a face-centered cubic structure, wherein each sphere contacts 12 others (six in the same layer, three in the layer above, and three in the layer below). Hence, the voids of the artificial opal have quasicubic and quasitetrahedral forms with concave sides, which are connected to each other by vertices. After filling in the voids a lacy free-standing opal-like structure appears.

In our previous work $^{35}$ polarized small-angle neutron scattering (SANS) was used to detect the transformation of the magnetic structure of three-dimensional Ni inverse OLSs under the applied field. Different contributions to the neutron scattering have been analyzed: the nonmagnetic one, the pure magnetic one, and the nuclear-magnetic interference. The patterns of the hysteresis loops of the pure magnetic and interference scattering intensities were different for different orientations of the magnetic field applied along two axes of the Ni OLSs: along the [12 1$]$ and [11 1$]$ axes. This is related to the geometrical shape of the inverse OLSs. In general, the experiment demonstrated that the three-dimensional (3D) geometrical shape of the structure led to a complex distribution of the magnetization in the sample, which is far from being simply uniform.

We have now performed a similar study of the Co inverse photonic crystal using a combination of polarized SANS and complementary techniques. The neutron scattering cross sections of materials made of $\mathrm{Ni}$ and $\mathrm{Co}$ show a valuable difference from each other. For $\mathrm{Ni}$ the nuclear scattering amplitude $A_{n}$ is much larger than the magnetic scattering amplitude $A_{m}$. This means that the magnetic scattering is hardly distinguishable because it is superimposed on the highly intensive reflections from the nuclear structure. ${ }^{35}$ Luckily, for Co the nuclear scattering amplitude $A_{n}$ is twice as small as the magnetic scattering amplitude $A_{m}$. In this case the magnetic contribution is much more intense while the nuclear contribution is very weak. This has allowed us to detect the magnetic transformations in the Co-based OLS in more detail than was possible for the Ni OLS.

The paper is organized in the following way. Sections II and III describe the synthesis and structural properties of the cobalt inverse OLS on the microscopic and atomic levels performed with use of microradian $\mathrm{x}$-ray diffraction and wideangle powder diffraction methods. The magnetic properties investigated by superconducting quantum interference device (SQUID) magnetometry and polarized SANS are presented in Sec. IV. The interpretation of the results and discussion of the magnetic transformation in such spatially ordered three-dimensional systems under an applied field are presented in Sec. V. Section VI gives short concluding remarks.

\section{SAMPLE}

The inverse Co OLS films were prepared using a templating technique. Colloidal crystal films (template) with an area of $10 \times 20 \mathrm{~mm}^{2}$ and a thickness of $10 \mu \mathrm{m}$ were grown on conductive substrates by the vertical deposition method from an aqueous suspension of monodisperse polystyrene microspheres with a diameter of $450 \pm 20 \mathrm{~nm}$ at $60^{\circ} \mathrm{C}$. $^{36}$ Electrochemical crystallization of cobalt in the voids between the spheres was carried out in a three-electrode cell at room temperature. The counterelectrode was a $\mathrm{Pt}$ wire and the reference was a saturated $\mathrm{Ag} / \mathrm{AgCl}$ electrode connected to the cell via a Luggin capillary. A solution of $0.2 M \mathrm{CoSO}_{4}$, $0.3 M \mathrm{H}_{3} \mathrm{BO}_{3}$, and $3.5 M \mathrm{C}_{2} \mathrm{H}_{5} \mathrm{OH}$ was used for potentiostatic Co deposition at $E_{d}=0.9 \mathrm{~V}$ versus the reference electrode. In order to obtain a free-standing Co structure on the substrate, the polystyrene microspheres were dissolved in toluene for $3 \mathrm{~h}$. An example of a scanning electron microscopy (SEM) image of the sample is shown in Fig. 1. The image shown in Fig. 1 can be clearly attributed to either the (111) plane of the fcc structure or the base plane of the random hexagonal close-packed (rhcp) structure.

Typically the vertical deposition method produces a thin OLS consisting of 10-40 layers on the substrate. The geometry of synthesis directly determines the geometry of the growing film crystal. The direction perpendicular to the substrate typically coincides with the [111] axis of the fcc structure. The vertical axis (axis of aqueous suspension drying or meniscus movement) corresponds to the [20ㄹ] crystallographic axis. Thus, one is able to have a clear understanding of the orientation of the crystal already at the stage of synthesis. The question remaining unsolved is always about the quality of the crystals obtained, which can degrade at each step of the

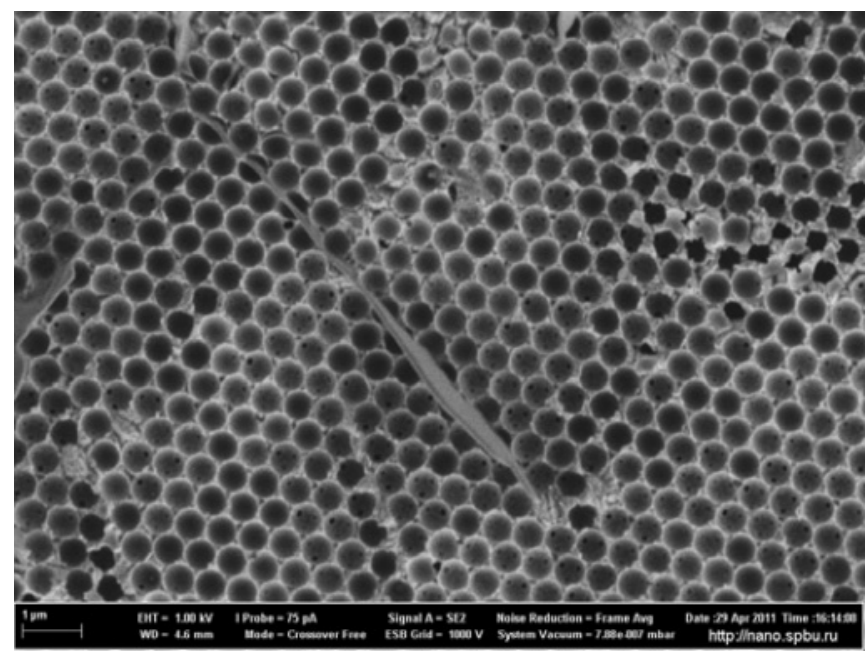

FIG. 1. SEM image of the top view of the Co inverse opal structure. 


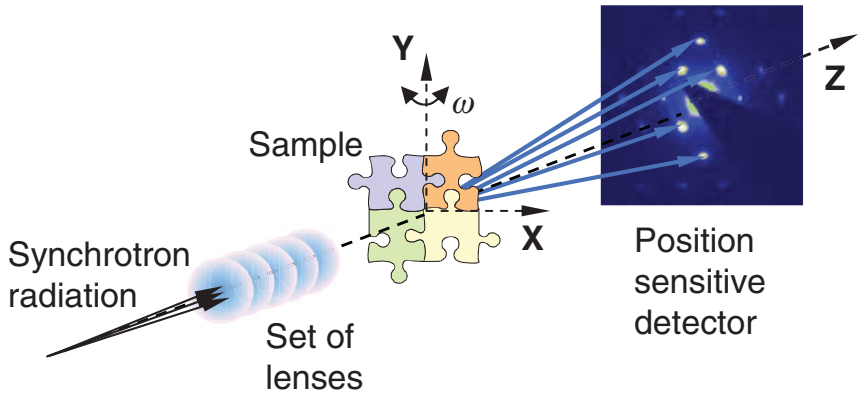

FIG. 2. (Color online) Schematic drawing of the diffraction experiment with the use of synchrotron radiation.

synthesis: (1) synthesis of the template, (2) synthesis of the inverse structure, and (3) solution of the template.

\section{STRUCTURAL PROPERTIES}

\section{A. Microradian x-ray diffraction}

Microradian x-ray diffraction experiments were performed at the DUBBLE BM26 line at the European Synchrotron radiation facility (ESRF), France (Fig. 2). We used a monochromatic x-ray beam of $\lambda=0.097 \mathrm{~nm}$, a wavelength spread of $\Delta \lambda / \lambda=2 \times 10^{-4}$, and size at the sample of $0.5 \times 0.5 \mathrm{~mm}^{2}$. Scattering was registered at $8 \mathrm{~m}$ distance by a two-dimensional CCD detector (Potonic Science, 4008 $\times 2671$ pixels of $\left.22 \mu \mathrm{m}^{2}\right) .^{37,38}$ The angular resolution of the setup was reduced to $10 \mu \mathrm{rad}$ due to the system of focusing beryllium compound refractive lenses installed just before the sample. Because of this resolution combined with the high brilliance of the synchrotron source, one is able to study large-scale periodic structures with period up to $5 \mu \mathrm{m}$. The typical exposure time was $1 \mathrm{~s}$. The sample was rotated around the [202] crystallographic axis in the range of $\omega \in\left[-65^{\circ}-+65^{\circ}\right]$, where $\omega=0$ corresponds to the geometry of the film surface
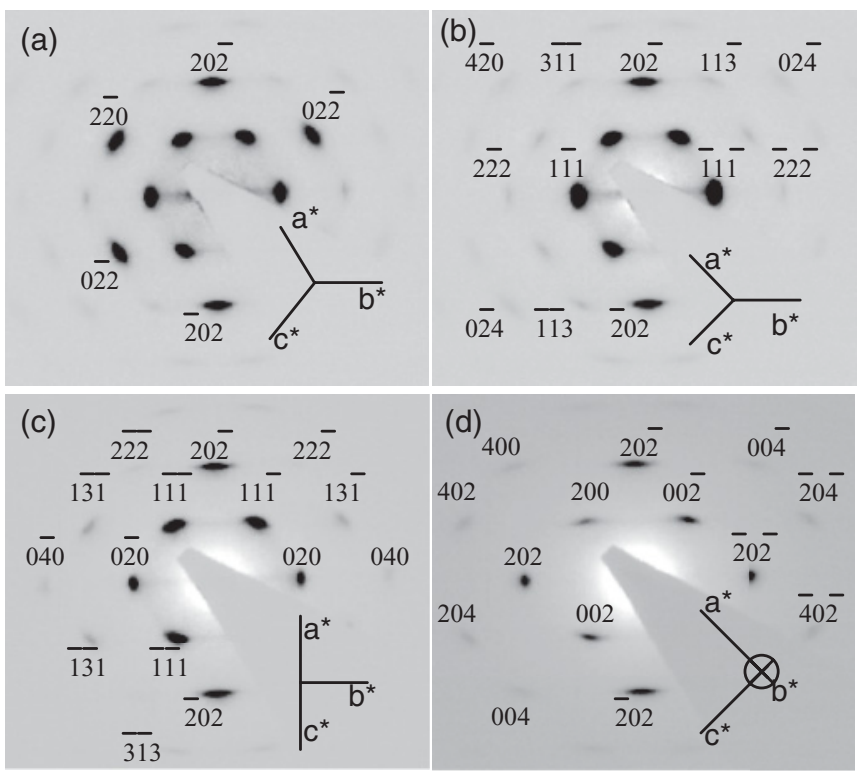

FIG. 3. Diffraction patterns of the Co OLS at various rotation angles $\omega=0^{\circ}$ (a), $19^{\circ}(\mathrm{b}),-35^{\circ}$ (c), and $55^{\circ}$ (d) around the [20र्2] axis.

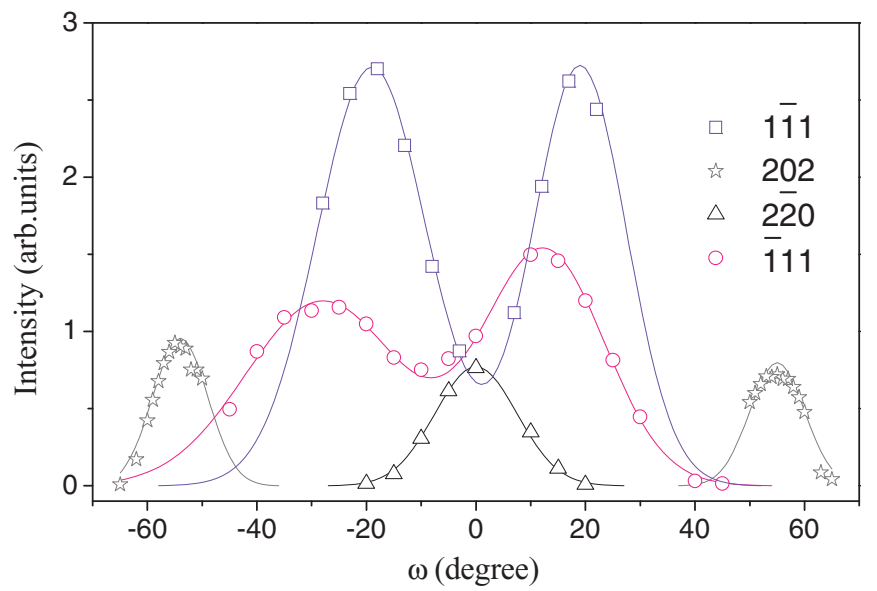

FIG. 4. (Color online) $\omega$ dependence of the integrated intensity for the reflections $1 \overline{1} 1,202,2 \overline{2} 0$, and $1 \overline{1} \overline{1}$.

perpendicular to the beam axis (Fig. 2). The results of the experiment with the inverse Co OLS are shown in Figs. 3 and 4.

Figures 3(a), 3(b), 3(c), and 3(d) show the diffraction patterns for the reciprocal lattice of the fcc structure cut by the (111), (121), (101), and (010) planes, respectively. Most of the Bragg peaks correspond to the fcc structure (indexed reflections) with the lattice constant of $640 \pm 10 \mathrm{~nm}$. Additional reflections can arise due to the stacking faults of the $A, B$, and $C$ close-packed hexagonal layers along the growth axis of the template (the [111] direction of the fcc structure)..$^{39,40}$

Figure 4 demonstrates the $\omega$ dependence of integral

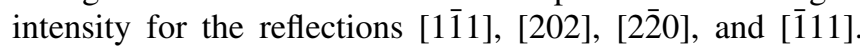
The presence of the symmetrical maxima at $\omega= \pm 19^{\circ}$ and $\pm 55^{\circ}$ gives evidence for the coexistence of the twinning planes. The $\omega$ scans for all reflections, shown in Fig. 4, have full widths at half Maximum of order which implies strong orientational disorder within the crystal. Moreover, for the [1111] reflection an additional contribution to the rocking curve width can originate from the stacking disorder present between the close-packed (111) planes.

In conclusion, these observations confirm that the electrochemical method of synthesis indeed allows one to duplicate the structure of artificial opals and to obtain the inverse OLS ordering in twin fcc structures with the lattice constant of $640 \pm 10 \mathrm{~nm}$ but also with a clear tendency to the random hexagonal close-packed structure along the [111] axes.

\section{B. Wide-angle powder diffraction}

The wide-angle powder $\mathrm{x}$-ray diffraction experiment was performed at the MAR diffractometer at the BM01A beamline (SNBL at the ESRF) with wavelength $\lambda=0.0717 \mathrm{~nm}$. The scattering pattern from the inverse Co OLS gives an example of typical powder diffraction, i.e., a system of concentric rings of equal intensity along a ring (Fig. 5, inset). The positions of reflections are well described by a superposition of the hcp and fcc atomic structures (Fig. 5). The content of these phases is 5\% fcc and 95\% hcp with lattice constants $a_{0}=3.55 \AA$ for the fcc structure and $a_{0}=2.51 \AA, c_{0}=4.07 \AA$ 


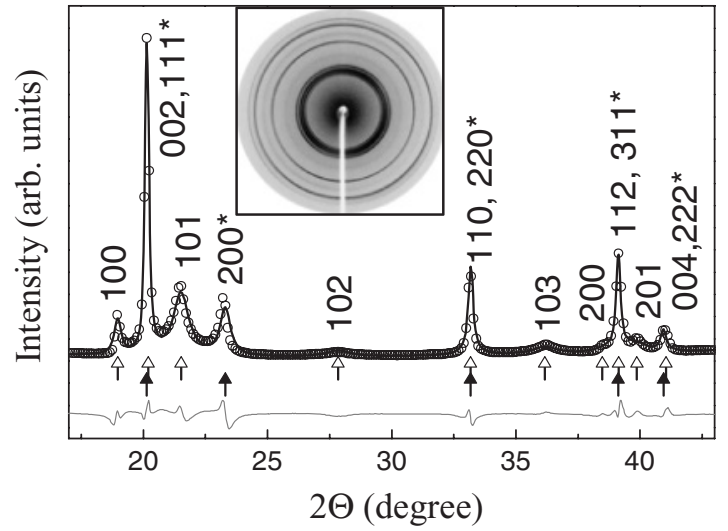

FIG. 5. X-ray powder diffractogram for the inverse Co OLS sample. The black arrows indicate the positions of fcc reflections, the open arrows the positions of hep reflections. The inset shows the powder diffraction pattern, i.e., the system of concentric rings.

for the hep structure. It is well known that bulk Co exists in two ferromagnetic thermodynamically stable phases, at ambient pressure, possessing different magnetic properties: the hcp phase or $\alpha$-Co below $388^{\circ} \mathrm{C}$, and the fcc phase or $\beta$-Co above $450{ }^{\circ} \mathrm{C}$. The coexistence of hcp and fcc phases at room temperature can be ascribed to the electrochemical synthesis of the OLS structure, which can lead to the mixture of phases during the formation of nanoparticles.

\section{MAGNETIC PROPERTIES}

\section{A. SQUID measurements}

The magnetic measurements were carried out on the MPMS-5S SQUID magnetometer. The magnetic field was applied either in the film plane of the inverse OLS sample (in plane) $\omega=0^{\circ}$, i.e., direction $\mathbf{H} \perp$ [111], or inclined to the film plane (out of plane) at the angle $\omega \neq 0^{\circ}$. Figure 6 shows the hysteresis loops of the magnetization for the inverse Co OLS measured at $300 \mathrm{~K}$ and taken at different orientations of the magnetic field with respect to the film plane. The magnetization is more or less fully saturated for the applied magnetic field $H=1 \mathrm{~T}$. The inset in Fig. 6 shows that the coercive field $H_{C}$ has a maximum at $\omega=60^{\circ}$. In contrast, the remanent magnetization $M_{R}$ monotonically decreases with increase of the inclination angle $\omega$. The maximal value of $M_{R}$ at the angle $\omega=0^{\circ}$ reaches $46 \%$ of the saturated magnetization $M_{s}$.

This is related to the anisotropic shape of the sample. Indeed, the thickness of the metallic layer $(10 \mu \mathrm{m})$ is $10^{3}$ times smaller than its length or width $\left(10 \times 20 \mathrm{~mm}^{2}\right)$. As a result, the film plane is the easy plane for magnetization with the hard axis perpendicular to it. It should be noticed that the measurements did not show a noticeable difference in the hysteresis loops of magnetization for the field applied in different directions within the sample plane.

\section{B. Polarized SANS technique}

Polarized SANS measurements were carried out with the instrument SANS-2 at the Geesthacht Neutron Facility (GeNF), Germany. A schematic drawing of the setup is shown in Fig. 7. The instrument is equipped with a polarizer

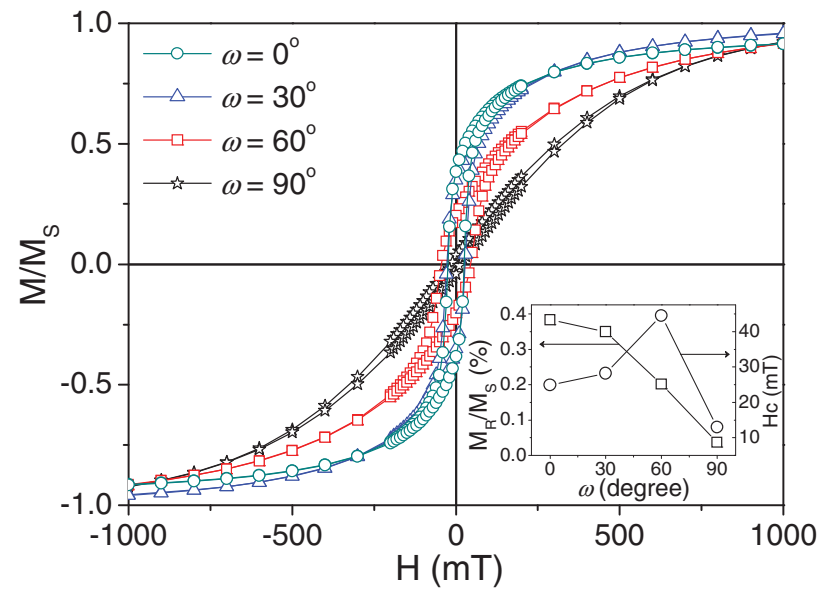

FIG. 6. (Color online) Hysteresis loops for the inverse Co OLS taken at different orientations of the magnetic field with respect to the film plane: the inclination angle $\phi=0^{\circ}, 30^{\circ}, 60^{\circ}, 90^{\circ}$.

and spin flipper to provide the possibility of studying the polarization-dependent neutron cross section. A polarized beam of neutrons with an initial polarization $P_{0}=0.95$, a neutron wavelength $\lambda=1.2 \mathrm{~nm}$, a bandwidth $\Delta \lambda / \lambda=0.1$, a divergence $\eta=0.5 \mathrm{mrad}$, and a size of $8 \times 8 \mathrm{~mm}^{2}$ was exploited. The scattered neutrons were detected by a positionsensitive detector with resolution of $256 \times 256$ pixels, each of $2.2 \times 2.2 \mathrm{~mm}^{2}$ size. The detector-sample distance was set at its maximum of $21.5 \mathrm{~m}$ that the $q$ range was covered from 0.005 to $0.07 \mathrm{~nm}^{-1}$ with a step of $0.0005 \mathrm{~nm}^{-1}$. An external magnetic field was applied perpendicular to the incident beam and ranged from -200 to $+200 \mathrm{mT}$. The polarization followed the direction of the magnetic field, $\mathbf{P}_{0} \| \mathbf{H}$. The data were taken at room temperature after the remagnetization process, i.e., after application of the opposite magnetic field of $200 \mathrm{mT}$. The typical counting time was of the order of $2 \mathrm{~h}$.

The total cross section of polarized neutrons for a magnetic structure with a large period can be split into three contributions: $\Sigma_{\text {tot }}=\Sigma_{N}+\Sigma_{M}+\Sigma_{I}$, where $\Sigma_{N}$ and $\Sigma_{M}$ are the nuclear and magnetic scattering cross sections, respectively, which are independent of the neutron polarization $\mathbf{P}_{0}$, whereas $\Sigma_{I}$ describes the nuclear-magnetic interference and is proportional to $\mathbf{P}_{0}{ }^{41}$ Each contribution for the magnetized sample

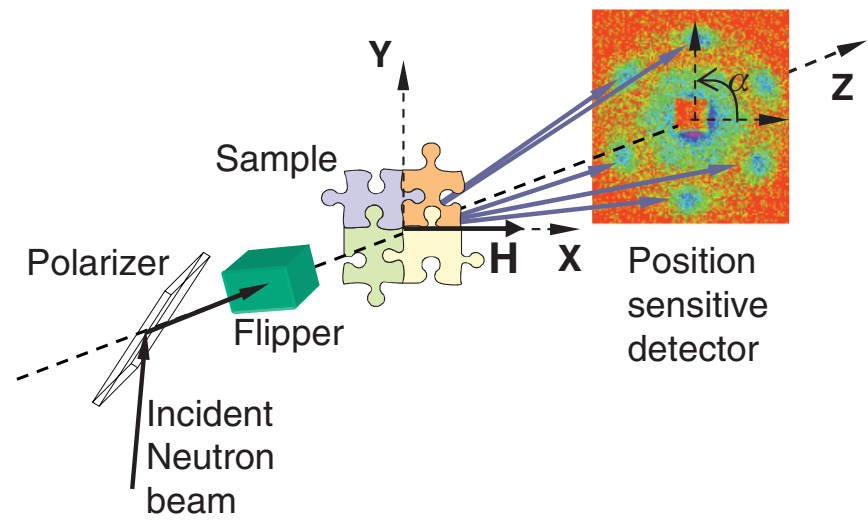

FIG. 7. (Color online) Schematic drawing of the polarized neutron diffraction experiment. 
is proportional to the product of the square of the structure factor $S(\mathbf{Q})$ - as a result of scattering from the structure - and the square of the form factor $F(Q)$ - as a result of scattering from the particle that is the base element of this structure. The form factor of the elements building an inverted structure is identical to the form factor of a spherical particle, and it can be written as follows: ${ }^{42}$

$$
F(Q)=\frac{\sin (Q R)-Q R \cos (Q R)}{(Q R)^{3}},
$$

where $R$ is the radius of the cavities in the inverse artificial opal.

In the simplest case of a fully magnetized sample we have for the three contributions

$$
\begin{gathered}
\Sigma_{N}=\left|A_{n} \mathcal{S}(\mathbf{Q}) F(Q)\right|^{2}, \\
\Sigma_{M}=\left|A_{m} \mathbf{m}_{\perp \mathbf{Q}} \mathcal{S}(\mathbf{Q}) F(Q)\right|^{2}, \\
\Sigma_{I}=2\left(\mathbf{P}_{0}\langle\mathbf{m}\rangle_{\perp \mathbf{Q}}\right) A_{n} A_{m}|\mathcal{S}(\mathbf{Q}) F(Q)|^{2},
\end{gathered}
$$

where $\mathbf{m}$ is the unit vector of the magnetization $\mathbf{M}$, and $\mathbf{m}_{\perp \mathbf{Q}}=\mathbf{m}-(\mathbf{Q m}) \mathbf{Q} / Q^{2}$. The nuclear and magnetic contributions are determined by the amplitudes $A_{n}=b_{\mathrm{Co}} N_{0}$ and $A_{m}=p_{\mathrm{Co}} N_{0}$, respectively. For the given case $b_{\mathrm{Co}}=0.25 \times$ $10^{-12} \mathrm{~cm}$ is the coherent scattering length of Co nuclei, $p_{\mathrm{Co}}=0.47 \times 10^{-12} \mathrm{~cm}$ is the coherent magnetic scattering length of the Co atom in the small-angle range, and $N_{0}$ is the atom density.

The neutron scattering intensities $I\left(\mathbf{Q},+P_{0}\right)$ and $I(\mathbf{Q}$, $-P_{0}$ ) were measured in the experiments with a neutron beam with polarization parallel and antiparallel to the external magnetic field, respectively. The polarization-independent part of the scattering represents the sum of the nuclear and magnetic scattering,

$$
I(\mathbf{Q})=\left[I\left(\mathbf{Q},+P_{0}\right)+I\left(\mathbf{Q},-P_{0}\right)\right] / 2 \sim I_{0} V_{0}\left(\Sigma_{N}+\Sigma_{M}\right),
$$

where $I_{0}$ is the incident beam intensity and $V_{0}$ is the sample volume. The pure magnetic coherent part is proportional to the difference between the magnetic cross sections of the sample in two principally different states: partially magnetized in the finite field $H$ and fully demagnetized at $H \sim H_{c}$, where $H_{c}$ is the coercive force:

$$
I_{M}(\mathbf{Q})=I(\mathbf{Q}, m(H))-I\left(\mathbf{Q}, m\left(H_{c}\right)\right) \sim I_{0} V_{0} \Sigma_{M} .
$$

The polarization-dependent part of the scattering is determined as

$$
\Delta I(\mathbf{Q})=\left[I\left(\mathbf{Q},+P_{0}\right)-I\left(\mathbf{Q},-P_{0}\right)\right] / 2 \sim I_{0} V_{0} \Sigma_{I} .
$$

It is attributed to the nuclear-magnetic interference, indicating the correlation between the magnetic and nuclear structures. Both $I_{M}(\mathbf{Q})$ and $\Delta I(\mathbf{Q})$ are completely free of the nuclear contribution. In Eqs. (5)-(7) we neglect the occurrence of the small-angle diffuse scattering caused by structure imperfections and concentrate on the Bragg reflections stemming from the scattering on the large-scale structure.

\section{Polarized SANS data}

The neutron diffraction patterns are shown in Fig. 8. The observed reflections correspond to neutron scattering
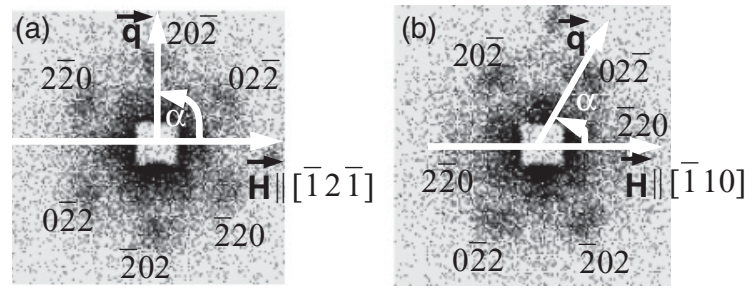

FIG. 8. Neutron scattering map for inverse Co OLS sample for two different geometries with (a) $\mathbf{H} \|[\overline{1} 2 \overline{1}]$ (geometry I) and (b) H $\|[\overline{1} 10]$ (geometry II) at $H=200 \mathrm{mT}$.

on the $\{202\}$ planes, that is in good agreement with the microradian X-ray diffraction data [Fig. 3(a)]. We investigated the influence of the structural anisotropy on the behavior of the magnetization vector in the reversal magnetization process: the external magnetic field was applied in two different geometries: geometry I, when $\mathbf{H}$ is along the [ $\overline{1} 2 \overline{1}]$ axis [Fig. 8(a)] and geometry II, when $\mathbf{H}$ is along the [110] axis [Fig. 8(b)]. In order to appropriately estimate the magnetic contribution $I_{m}(\mathbf{Q})$ and interference contribution $\Delta I(\mathbf{Q})$, one has to analyze these intensities as a function of the azimuth angle $\alpha$, where $\alpha$ is the angle between the direction of the scattering vector $\mathbf{Q}$ and the field $\mathbf{H} \| P_{0}$ direction, which points along the horizontal in the detector plane (Fig. 7).

Figure 9 shows the intensity $I(Q, \alpha)$ at $Q=0.024 \mathrm{~nm}^{-1}$ as a function of the azimuthal angle $\alpha$ for the sample magnetized along $\mathbf{H} \|[\overline{1} 2 \overline{1}]$ [Fig. 9(a)] and $\mathbf{H} \|[\overline{1} 10]$ [Fig. 9(c)]. For geometry I [Fig. 9(a)], we observe six maxima of equal intensity at $60^{\circ}$ intervals, which correspond to the six 202-type reflections. These six reflections are visible in Fig. 8(a) for the magnetized sample only and, therefore, have magnetic origin. The intensity, shown in Fig. 9(a), consists of three contributions: a diffuse background $\left(I_{b g}\right)$ given by the horizontal dashed line and the sum of the nuclear and magnetic reflections given by scattering on $\{202\}$ planes with the ratio between nuclear and magnetic contributions in intensities of 1 to 3, approximately, for the magnetized sample. The equal intensities of the magnetic reflections (independent of the mutual orientations of $\mathbf{H}$ and $\mathbf{Q}$ ) show that the magnetization inside the unit element of the OLS is not aligned along the field axis but rather subject to the structural anisotropy of the sample. This will be discussed in more detail in Sec. V.

The picture of scattering is similar for geometry II [Fig. 9(c)] but on the qualitative level only. In particular, the intensities of the two reflections $\overline{2} 20$ and $2 \overline{2} 0$ at $\alpha=0^{\circ}$ and $\alpha=180^{\circ}$, respectively, become so small that they are hardly visible. These two reflections with $\mathbf{Q} \| \mathbf{H}$ are suppressed by the magnetic field, showing domination of the magnetic coherent scattering over its nuclear counterpart for the other clearly visible reflections. The remaining part of the intensity at $\alpha=0^{\circ}$ and $\alpha=180^{\circ}$ can be referred to the nuclear Bragg reflections imposed on the top of the considerable background level (70 counts $/ \mathrm{h})$.

The angular dependence of the polarization-dependent part, $\Delta I(\alpha)$, at $Q$ corresponding to 202 reflections is presented in Figs. 9(b) and 9(d), for two different geometries. The positions of the maxima coincide with those of the polarizationindependent part in Figs. 9(a) and 9(c). According to Eqs. (3) and (4), the magnetic term is proportional to $\left|\mathbf{m}_{\perp \mathbf{Q}}\right|^{2}$ while the 

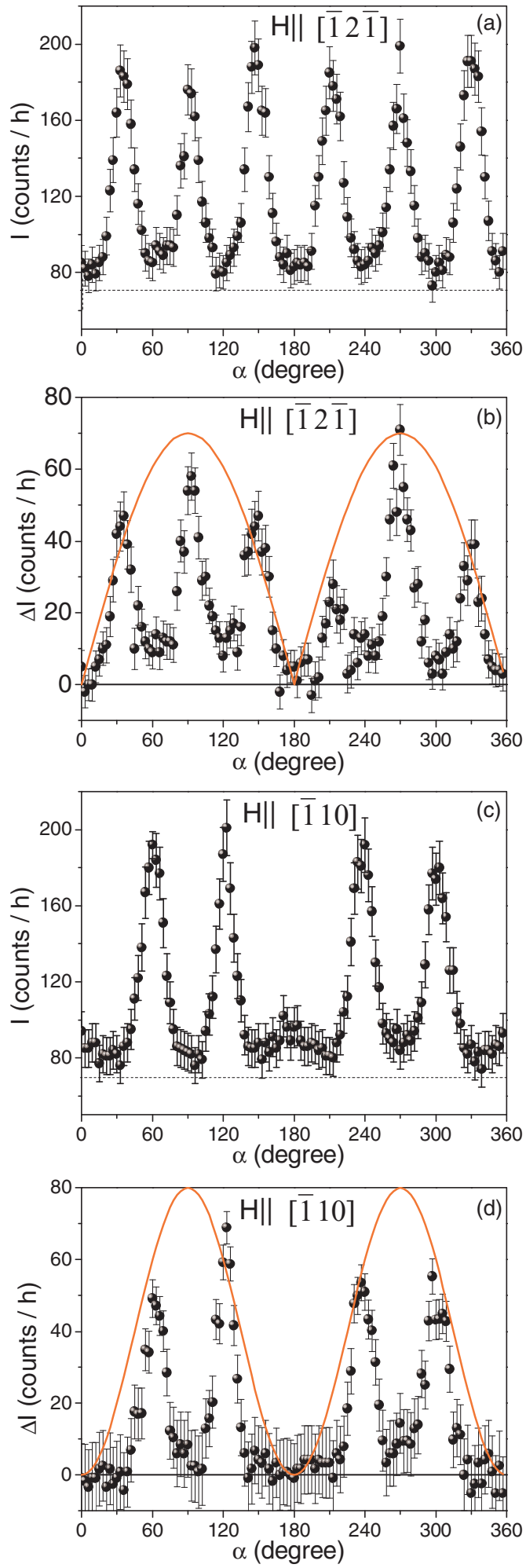

FIG. 9. (Color online) $\alpha$ dependence of the polarizationindependent intensity $I$ and polarization-dependent intensity $\Delta I$ for the $20 \overline{2}$ reflections for the external magnetic field $H=200 \mathrm{mT}$

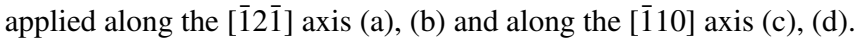
The lines in (b) and (d) represent schematically the $|\sin \alpha|$ dependence and $|\sin \alpha|^{2}$ dependence, respectively.

interference term is proportional to $\left(\mathbf{P}_{0}\langle\mathbf{m}\rangle_{\perp \mathbf{Q}}\right)$. For the fully magnetized sample both contributions should follow a $\sin ^{2} \alpha$ dependence. This is fulfilled for geometry II [Fig. 9(d) (solid curve)], but is clearly not the case for geometry I [Fig. 9(b)]. For the latter case the magnetic intensity is $\alpha$ independent [Fig. 9(a)] while the simple $|\sin \alpha|$ dependence plotted in Fig. 9(b) gives a better description than the theoretically predicted dependence. The ratio of interferences and the total intensities can be estimated for the fully magnetized sample at $\alpha=90^{\circ}$ :

$$
\frac{\Sigma_{I}}{\Sigma_{N}+\Sigma_{M}}=2 P_{0} \frac{p_{\mathrm{Co}} b_{\mathrm{Co}}}{p_{\mathrm{Co}}^{2}+b_{\mathrm{Co}}^{2}} \approx 0.8 P_{0} .
$$

For geometry $\mathrm{I}$ the observed ratio $\Delta I /\left(I-I_{b g}\right) \approx 0.5$ is somewhat lower than we estimated using Eq. (8). This also suggests that the sample is not fully magnetized. As will be shown below, the system under study can be well described by the model, when different parts of the regular structure show different degrees of magnetic ordering with the magnetization pointing in different directions.

\section{Polarized SANS: Q dependence}

Our preliminary analysis in Sec. IVC shows that the polarization-dependent part of the scattering $\Delta I(Q)$ follows the $\sin ^{2} \alpha$ dependence for geometry II and $|\sin \alpha|$ dependence for geometry I. In other words, the sample is not completely magnetized for geometry I and, probably, better magnetized for geometry II for the same value of the magnetic field of $200 \mathrm{mT}$. For a quantitative analysis the data were plotted as a function of the momentum transfer $Q$ for certain $Q$ directions along the $[20 \overline{2}]$ axes. The intensity was averaged over the angle range of $\Delta \alpha=10^{\circ}$. Figure 10 shows an example of the $Q$ dependence of the neutron scattering intensity (a) for the polarization-independent part $I(Q)$ [Eq. (5)], (b) for the magnetic-field-dependent part $I_{M}(Q)$ [Eq. (6)], and (c) for the polarization-dependent part $\Delta I(Q)$ [Eq. (7)] at $H=200 \mathrm{mT}$ and $\alpha=90^{\circ}$ taken for geometry $\mathrm{I}$. The position of the reflection in $Q$ space corresponds very well to the $20 \overline{2}$ Bragg reflection of the fcc structure with $a_{0}=640 \pm 10 \mathrm{~nm}$, which is in good agreement with electron microscopy and microradian x-ray diffraction data.

The polarization-independent intensity $I(Q)$ [Fig. 10(a)] contains four contributions: coherent nuclear and magnetic contributions, as well as incoherent (diffuse) nuclear and magnetic ones. The coherent contributions give rise to the Bragg reflection at $Q=0.024 \mathrm{~nm}^{-1}$. The incoherent contributions give rise to the intensity at very small values of the momentum transfer $Q<0.018 \mathrm{~nm}^{-1}$.

Figure 10(b) shows the magnetic scattering $I_{m}(Q)$ obtained using Eq. (6). In Eq. (6) the component $I(Q, m(H))$ gives the coherent magnetic scattering at $H=200 \mathrm{mT}$. It can be described in the fit by a sum of Gaussians for every Bragg peak with the positions corresponding to the fcc structure. In Eq. (6) the component $I\left(Q, m\left(H_{c}\right)\right)$ of the cross section at $H=H_{c}$ is the diffuse scattering from the domain structure and is described by the squared Lorentzian $I(Q)=A_{D} /\left(Q^{2}+\right.$ $\left.\kappa^{2}\right)^{2}$, where $\kappa$ is the inverse correlation length, which does not depend on the magnetic field and is equal to $0.016 \mathrm{~nm}^{-1}$. Thus, the magnetic scattering $I_{m}(Q)$ is free of the nuclear contributions and consists of the positive contribution to the magnetic Bragg reflections and the negative contribution to the 

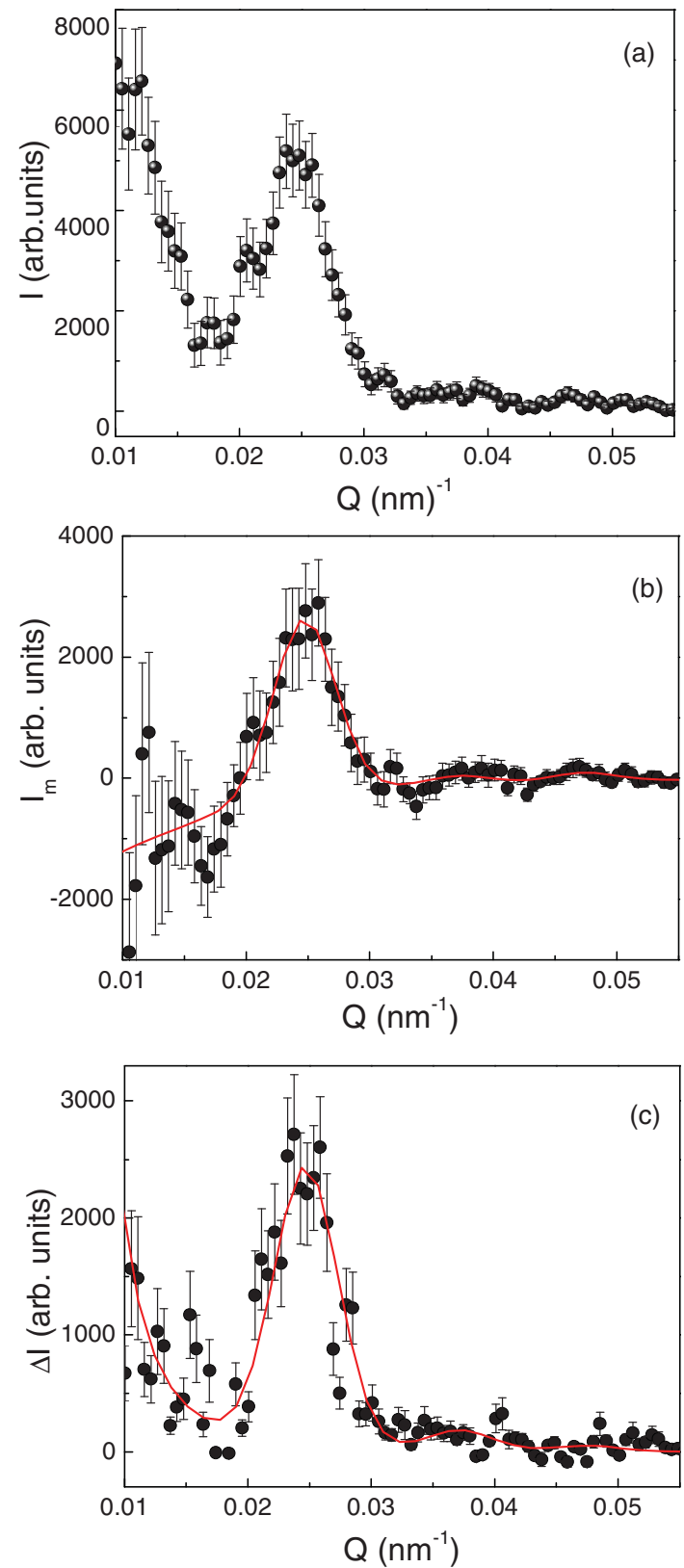

FIG. 10. (Color online) Momentum transfer dependence of (a) the total intensity of the neutron scattering, $I(Q)$, (b) the magneticfield-dependent intensity $I_{m}(Q)$, and (c) the interference intensity $\Delta I(Q)$ at $H=200 \mathrm{mT}$.

magnetic diffuse scattering coming from the magnetic largescale domains and inhomogeneties. The solid line in Fig. 10(b) is the result of the fit, which gives the amplitude of the Bragg reflection $C_{M}$ and the amplitude of the diffuse scattering $A_{D}$. The obtained parameters are shown in Figs. 11(b), 11(c), 12(b), 12(c), 13(b), and 13(c) as a function of the external magnetic field for different type of reflections in different geometries.

The graph in Fig. 10(c) gives the interference contribution to the reflection at the same $Q$ position as for the magnetic and nuclear scatterings. This shows that the nuclear structure is well correlated with the magnetic one in those parts of the OLS which are more or less magnetized. Although the graphs in Figs. 10(b) and 10(c) look quite similar, it is shown below
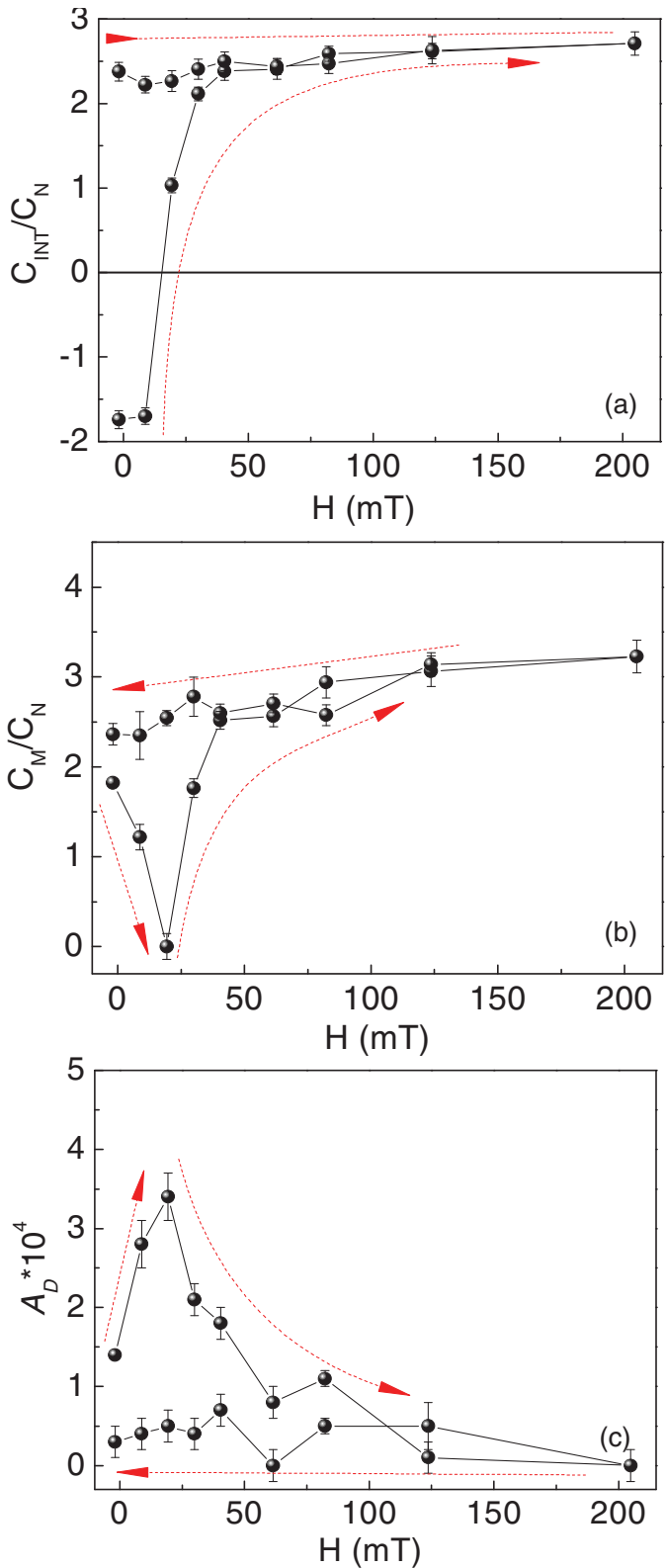

FIG. 11. (Color online) Field dependence of the ratios of interference contribution $C_{\mathrm{INT}} / C_{N}$ (a), magnetic contribution $C_{M} / C_{N}(\mathrm{~b})$, and magnetic diffuse contribution $A_{D}$ (c), normalized to the nuclear contribution, for the $20 \overline{2}$-type reflections $\left(\alpha=90^{\circ}\right)$ for geometry I.

that the purely magnetic and interference contributions give different and complementary information about the magnetic structure. The solid line in Fig. 10(c) is the result of the fit which gives the amplitude of the interference term $C_{\mathrm{INT}}$ at $Q=0.024 \mathrm{~nm}^{-1}$.

All reflections of 202 type visible in Fig. 8(a) (geometry I) can be divided into subgroups: reflections $20 \overline{2}$ and $\overline{2} 02$ with the angle of $90^{\circ}$ between $\mathbf{Q}$ and $\mathbf{H}$; and reflections $02 \overline{2}$, $0 \overline{2} 2,2 \overline{2} 0$, and $\overline{2} 20$ with the angle of $30^{\circ}$ between $\mathbf{Q}$ and $\mathbf{H}$. To improve the statistics, the intensities of these magnetic reflections were averaged over the subgroup. A similar action was taken for geometry II [Fig. 8(b)]. The further analysis will be done in terms of the parameters obtained from the fit: 

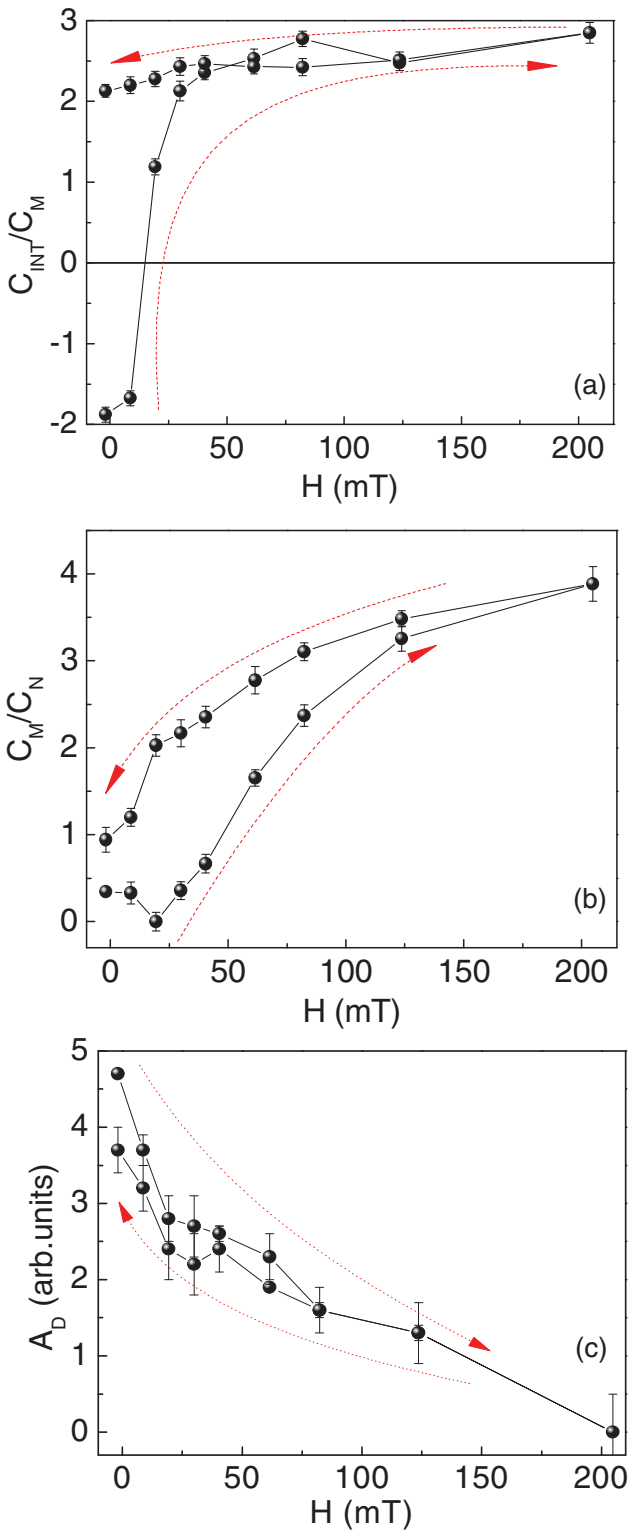

FIG. 12. (Color online) Field dependence of the ratios of interference contribution $C_{\mathrm{INT}} / C_{N}$ (a), magnetic contribution $C_{M} / C_{N}$ (b), and magnetic diffuse contribution $A_{D}$ (c), normalized to the nuclear contribution, for the $02 \overline{2}$-type reflections $\left(\alpha=30^{\circ}\right)$ for geometry I.

$C_{M}$ for the intensity of the magnetic Bragg reflection, $C_{\mathrm{INT}}$ for the intensity of the interference reflection, and $A_{D}$ for the intensity of the diffuse scattering. The normalized parameters are plotted as a function of the field for the Bragg $20 \overline{2}$ and $02 \overline{2}$ reflections for geometry I (Figs. 11 and 12 , respectively) and Bragg reflection $02 \overline{2}$ for geometry II (Fig. 13). In order to control the value for the magnetization on an absolute scale, the parameters obtained in the fit were normalized to the intensity $C_{N}$ of the nuclear Bragg reflection from the OLS.

\section{E. Polarized SANS: Field dependence}

As described in the theory [see Eqs. (6) and (7)] and realized in experiment [see Figs. 9(b) and 9(d)], the interference and magnetic contributions equal 0 at $\alpha=0^{\circ}, 180^{\circ}$ in the saturating
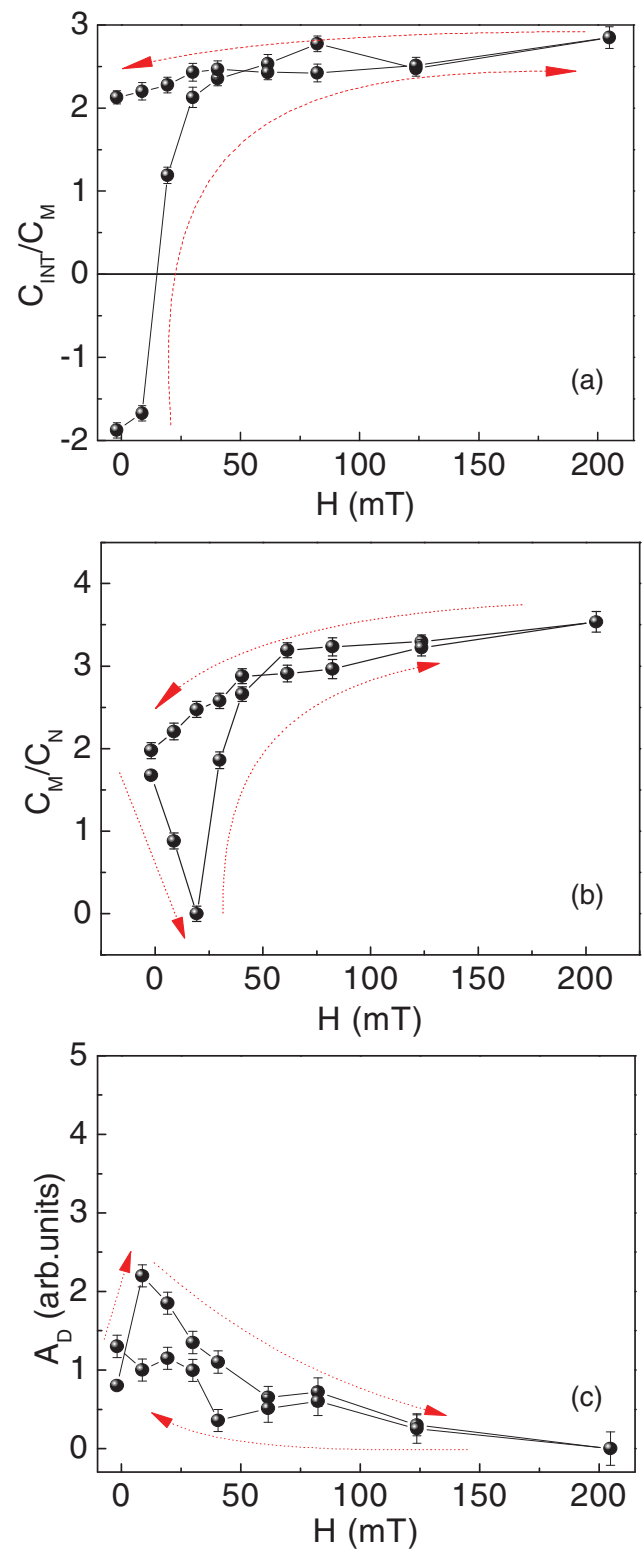

FIG. 13. (Color online) Field dependence of the ratios of interference contribution $C_{\mathrm{INT}} / C_{N}$ (a), magnetic contribution $C_{M} / C_{N}$ (b), and magnetic diffuse contribution $A_{D}$ (c), normalized to the nuclear contribution, for the $02 \overline{2}$-type reflections $\left(\alpha=60^{\circ}\right)$ for geometry II.

magnetic field $\left(\langle\mathbf{m}\rangle_{\perp \mathbf{Q}}\right)$. This means that the diffraction peak at $\alpha=0^{\circ}, 180^{\circ}$ has only the nuclear contribution to the neutron scattering and its intensity can be used as the value for the normalization $C_{N}$. One estimates that for the ideal case of a fully magnetized sample the ratio $\left(C_{M} / C_{N}\right)$ cannot exceed the value $\left(p_{\mathrm{Co}} / b_{\mathrm{Co}}\right)^{2}=3.53$ and the ratio $\left(C_{\mathrm{INT}} / C_{N}\right)$ cannot be higher than the value of $2 P_{0}\left(p_{\mathrm{Co}} / b_{\mathrm{Co}}\right)=3.76 P_{0} \approx 3.57$. Thus, if the system is homogeneously magnetized we should have the following ratio between the peak intensities of both the pure magnetic and the interference contributions: $I(\alpha=$ $\left.90^{\circ}\right): I\left(\alpha=60^{\circ}\right): I\left(\alpha=30^{\circ}\right): I\left(\alpha=0^{\circ}\right)=1: 0.75: 0.25: 0$.

The field dependence of the interference contribution $C_{\mathrm{INT}} / C_{N}$, the magnetic contribution $C_{M} / C_{N}$, and the magnetic diffuse contribution $A_{D}$ for the $20 \overline{2}$ reflection for 
geometry $\mathrm{I}\left(\alpha=90^{\circ}\right)$ is shown in Fig. 11. The interference contribution $C_{\mathrm{INT}} / C_{N}$ is proportional to the average magnetization projected onto the field axis $(\mathbf{m H})$ [see Eq. (4) for details]. It is clear from Fig. 11(a) that the value of $C_{\mathrm{INT}} / C_{N}$ tends to saturate in magnetic fields of $H>30 \mathrm{mT}$ and exhibits a magnetic hysteresis with the coercive force $H_{c}=17 \pm 3 \mathrm{mT}$ that corresponds to the demagnetized state of the sample. This hysteresis curve is analogous to that taken by SQUID for the sample in this geometry I (Fig. 6). However, one can see that the shapes of these two curves are different: the squareness of the hysteresis loops $M_{R} / M_{S}$ for the SQUID curve is 0.36 , while it is equal to 0.66 with respect to the possible maximum of $C_{\text {INT }} / C_{N}=3.57$ for the $20 \overline{2}$ reflection [Fig. 11(a)]. The values of the coercive force $H_{c}$ are also different: $12 \mathrm{mT}$ for SQUID and $17 \mathrm{mT}$ for $C_{\mathrm{INT}} / C_{N}$. It is interesting to note that the magnetic contribution $C_{\mathrm{INT}} / C_{N}$ is $75 \%$ saturated in a field of $200 \mathrm{mT}$, similarly to the SQUID magnetization. The observed differences reflect the fact that SQUID gives the value of the magnetization integrated over the whole $Q$ space, while the method of polarized neutron diffraction gives a differentiated value at a well-defined point of the $Q$ space.

The magnetic contribution $C_{M} / C_{N}$ demonstrates a hysteresis behavior with a clearly established minimum at $H_{c}$ pointing out the demagnetized state of the OLS structure [Fig. 11(b)]. Both the hysteresis and the minimum in the magnetic coherent scattering $C_{M} / C_{N}$ are well correlated with the hysteresis and maximum of the diffuse contribution $A_{D}$ [Fig. 11(c)]. This gives additional credibility to the model used in the treatment of the data and described above. Indeed, the maximum of the diffuse scattering corresponds to the appearance of magnetic domains, while the minimum of the magnetic diffraction peak corresponds to the decay of the coherent magnetic structure. These two processes should obviously be well correlated. Since the magnetic structure is destroyed at $H_{c}$, there is no interference between magnetic and nuclear structures at this value of the magnetic field. The magnetic behavior of the three parameters $\left(C_{\mathrm{INT}}, C_{M}, A_{D}\right)$ is self-consistent. Additionally, the SQUID magnetization curve is $75 \%$ saturated in a field of $200 \mathrm{mT}$, while for the $C_{M} / C_{N}$ curve it seems to exceed $90 \%$ of its maximal value of 3.53 .

Both the comparison to the SQUID measurements and the comparison to the expected values of the normalized neutron cross sections lead to the conclusion that the $\{202\}$ planes and the magnetic structure as a whole are not homogeneously magnetized. In other words, the direction of the magnetization in this sample strongly deviates from the direction of the external magnetic field but these deviations are regular in space and determined by the symmetry axes of the OLS.

The same distribution of the magnetization in the sample for geometry I gives the scattering to the $02 \overline{2}$ Bragg reflection. However, both the field-dependent behavior of the scattered intensities and the values achieved at the maximal field of $200 \mathrm{mT}$ are different. Figure 12 shows the field dependence of the ratios $C_{\mathrm{INT}} / C_{N}, C_{M} / C_{N}$, and $A_{D}$ for the $02 \overline{2}$-type reflections for geometry I $\left(\alpha=30^{\circ}\right)$. The interference contribution $C_{\text {INT }} / C_{N}$ [Fig. 12(a)] tends to saturate in magnetic fields of $H>50 \mathrm{mT}$, although it exhibits a magnetic hysteresis over the whole range of measured fields up to its highest value of $200 \mathrm{mT}$. The coercive field $H_{c}=15 \pm 3 \mathrm{mT}$ determines a fully demagnetized state. The interference curve $\left(C_{\mathrm{INT}} / C_{N} \approx 1.5\right)$ seems to be saturated and is twice as big as the possible maximum of $\left(C_{\mathrm{INT}} / C_{N}\right) \sin ^{2} \alpha=3.57 / 4=0.88$ for the $02 \overline{2}$ reflection [Fig. 12(a)].

The magnetic contribution $C_{M} / C_{N}$ also shows a hysteretic behavior [Fig. 12(b)] but a different type than for the $20 \overline{2}$ reflection [Fig. 11(b)]. The minimum intensity is observed in the whole range $H<H_{c}$ indicating the demagnetized state of the OLS. The intensity goes up as the magnetic field increases, showing no saturation up to the field of $200 \mathrm{mT}$. This is also correlated with the intensity of the diffuse scattering $A_{D}$ [Fig. 12(c)]. The diffuse intensity is maximal at $H=0$ and decreases with increasing external magnetic field in order to disappear at $H \geqslant 200 \mathrm{mT}$. The saturation is hardly observed in $C_{M} / C_{N}$ and $A_{D}$ for $02 \overline{2}$ reflections $\left(\alpha=30^{\circ}\right)$. This means that magnetic structure contributions to the $02 \overline{2}$ reflections are not magnetized up to the maximal field (200 mT).

A similar analysis of the data was conducted for geometry II (Fig. 8(b)). Figure 13 shows the field dependence of the ratios $C_{\text {INT }} / C_{N}, C_{M} / C_{N}$, and $A_{D}$ for the $02 \overline{2}$-type reflections for geometry II $\left(\alpha=60^{\circ}\right)$. The interference contribution $C_{\mathrm{INT}} / C_{N}$ [Fig. 13(a)] is saturated in magnetic fields of $H>30 \mathrm{mT}$ upon increase of the magnetic field and remains saturated upon its decrease down to $H=0$. The coercive field $H_{c}=16 \pm 3 \mathrm{mT}$ and the squareness of the hysteresis loops is equal to 0.75 with respect to the possible maximum of $\left(C_{\mathrm{INT}} / C_{N}\right) \sin ^{2} \alpha=$ $3.57 \times(3 / 4)=2.75$ for the Bragg reflections in geometry II.

The magnetic contribution $C_{M} / C_{N}$ also shows a hysteretic behavior [Fig. 13(b)] correlated to the intensity of the diffuse scattering $A_{D}$ [Fig. 13(c)]. The saturation is clearly observed for $C_{M} / C_{N}$ above $H=30 \mathrm{mT}$. This means that magnetic structure contributions to the Bragg reflections are magnetized up to the maximal field $(200 \mathrm{mT})$. The diffuse intensity is found maximal at $H \approx 10 \mathrm{mT}$ and decreases smoothly with increasing external magnetic field.

The magnetic and interference contributions do not appeared for the reflection $(\overline{2} 20)$ in geometry II, i.e., when the field is applied parallel to the scattering vector $\mathbf{Q}_{(\overline{2} 20)}$. This is expected because both for magnetized and nonmagnetized samples these contributions must be absent. In general, the intensity of the magnetic reflections shows the complexity of the local magnetic structure caused by the anisotropy of the inverse OLS.

\section{INTERPRETATION}

To interpret the obtained results one should make two important assumptions.

(i) The sample of OLS demonstrates clearly easy-plane anisotropy (111) caused by the very fact that the sample is a film with a thickness of the order of $10 \mu \mathrm{m}$ and an area of the order of $200 \mathrm{~mm}^{2}$. This is confirmed by the SQUID measurements, where the in-plane applied field magnetizes the sample much more easily than the out-of-plane magnetic field (Fig. 6).

(ii) The inverse Co OLS dictates the structural anisotropy and imposes well-defined limitations on the distribution of the magnetization over the sample. The crystallographic structure of the inverse OLS is built from a unit element consisting of three parts: a quasitetrahedron, a quasicube, and another quasitetrahedron. They are connected by vertices along the 


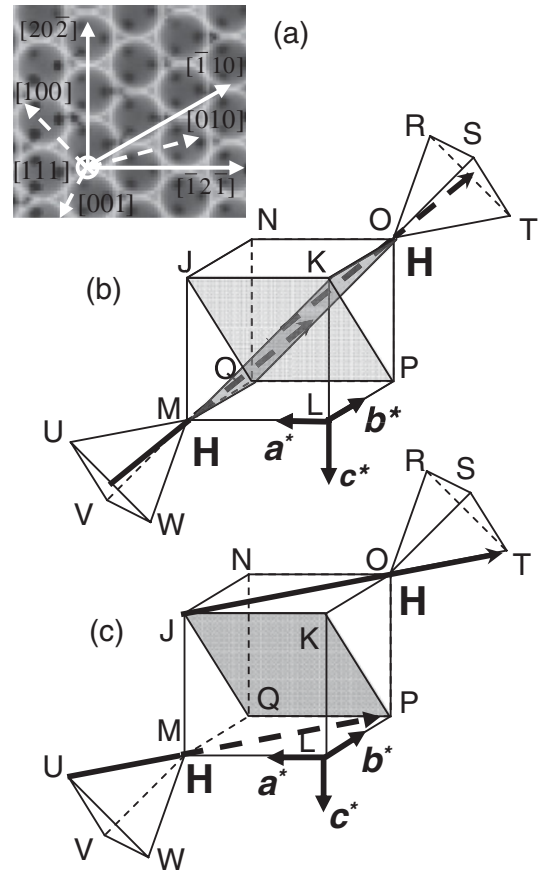

FIG. 14. Unit element of the magnetic structure of the inverse OLS with two different orientations of the in-plane applied field: $\mathbf{H} \|[\overline{1} 2 \overline{1}]$ (a) and $\mathbf{H} \|[\overline{1} 10]$ (b). The reflecting planes (202) and $(02 \overline{2})$ are shown on the picture by the shaded planes.

four [111]-type axes (Fig. 14). For an OLS with a period of $640 \mathrm{~nm}$ one can estimate that the central part has a "body" with a side $a \approx 174 \mathrm{~nm}$ and legs (eight for a cube and four for a tetrahedron) with a length of $l \approx 87 \mathrm{~nm}$. The tetrahedra are constituted by the $\{111\}$ planes with their edges coinciding with [110], [011], and [101] axes. The cubic axes have the same orientation as the basis of the colloidal crystal template $\left(\mathbf{a}^{*}, \mathbf{b}^{*}, \mathbf{c}^{*}\right)$. A schematic presentation of a layer of an inverse opal-like structure is shown in Fig. 15.

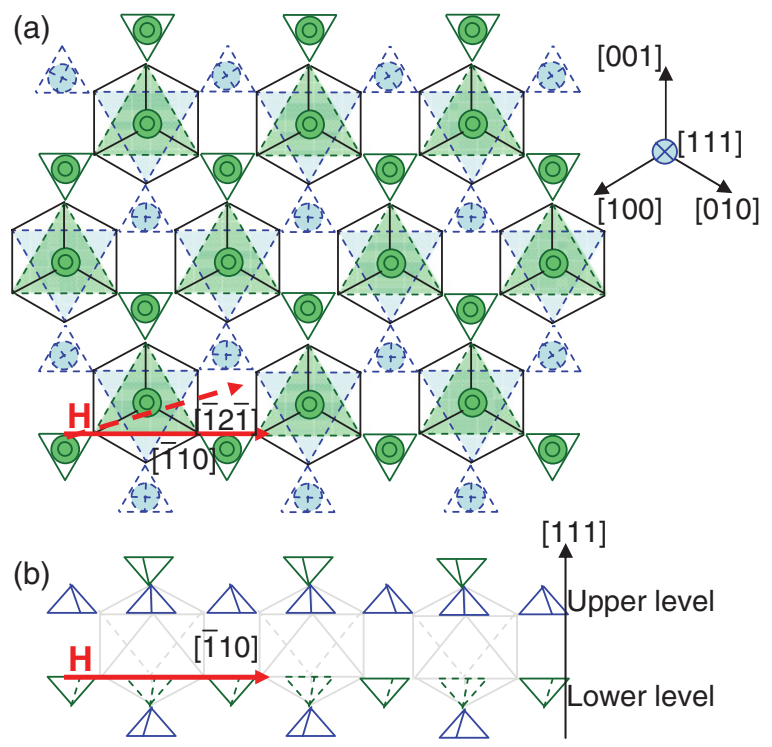

FIG. 15. (Color online) Schematic presentation of a layer of the inverse opal-like structure: top view (a) and side view (b).

\section{A. Model for magnetization}

We assume that the local magnetization is greatly influenced by the shape anisotropy of the structural units forming the structure. In particular, since at low and intermediate applied fields the magnetic field lines must be enclosed inside the ferromagnetic material of the OLS, the magnetization can only be directed along the pieces of magnetic material (legs) connecting the bodies. The magnetization passes through the unit element of the inverse OLS via the legs connecting quasitetrahedra and quasicubes. These leg connections constitute the easy axes for the magnetization inside the OLS sample, which are the four [111]-type axes. In reality, we should consider in the magnetization process with an in-plane applied field the three easy-magnetization axes only: [11 1$]$, [111], and [111]]. Note that the [111] axis is the hard axis for the macroscopically averaged sample magnetization for the film geometry of the sample.

Indeed, with the in-plane applied field, the legs connecting the samples in the [111] direction, perpendicular to the film plane, do not transmit any magnetization and, therefore, have random magnetization directions. Thus, on average they serve as "magnetically dead" elements. The sample, therefore, can be divided into a number of individual magnetic layers in the [111] direction, which are magnetized independently of each other. A schematic representative of the individual layer is shown in Figs. 15(a) (top view) and 15(b) (side view).

The layer decoupling gives the possibility of modeling in a simple way the magnetization process for an in-plane applied field. As shown in Fig. 15(b), the quasitetrahedra dislocate into two levels, the upper level with one leg pointing up and the lower level with one leg pointing down. These legs pointing up and down are parallel to the [111] axis of the OLS and as was mentioned above they can be considered as magnetically dead connections. The other quasitetrahedra realize links between the quasicubes via the other six vertices: three of them on the upper level and the other three on the lower level. For geometry I the distribution of magnetization inside the legs can be followed in Fig. 15. It is seen that one type of leg is almost parallel to the field, for example, in the [111] direction, while the other types of legs (with [ $\overline{1} \overline{1} 1]$ and [1 $\overline{1} \overline{1}]$ directions) are inclined at a large angle to the field axis. This leads to obvious consequences. The first type of leg connection is easily magnetized since the magnetic lines in them are almost parallel to the field. The second type is relatively difficult to magnetize and the magnetization in these legs deviates strongly from

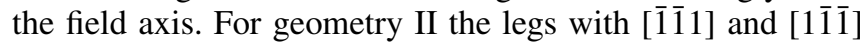
directions are inclined at a sharp angle to the field, while the other two types of leg are perpendicular to the field axis. This leads again to obvious consequences. The first type of leg connection is magnetized while the second type is not magnetized at all.

\section{B. Model for scattered intensities}

The neutron scattering cross section from an inverse Co OLS magnetized structure can be easily calculated from Eqs. (6) and (3) for the pure magnetic part and Eqs. (7) and (4) for the interference part on the assumption that the magnetization of different parts of the structural unit element can only be 
along the three low-index crystallographic directions (of the [001], [011], and [111] types). For geometry I, the distribution of magnetization inside the unit element is shown in Fig. 14(b) and can be written for the $20 \overline{2}$ Bragg reflection as

$$
\begin{aligned}
I_{M}(20 \overline{2}) \sim & V_{[\overline{1} 1 \overline{1}]} m_{[\overline{1} 1 \overline{1}]}^{2}+V_{[\overline{1} 0 \overline{1}]} m_{[\overline{1} 0 \overline{1}]}^{2}+V_{[010]} m_{[010]}^{2} \\
& +V_{[01 \overline{1}]} m_{[01 \overline{1}]}^{2} \cos ^{2} 30^{\circ}+V_{[\overline{1} 10]} m_{[\overline{1} 10]}^{2} \cos ^{2} 30^{\circ} \\
& +V_{[\overline{1} 00]} m_{[\overline{1} 00]}^{2} \cos ^{2} 45^{\circ}+V_{[00 \overline{1}]} m_{[00 \overline{1}]}^{2} \cos ^{2} 45^{\circ} \\
& +V_{[\overline{1} 11]} m_{[\overline{1} 11]}^{2} \cos ^{2} 55^{\circ}+V_{[11 \overline{1}]} m_{[11 \overline{1}]}^{2} \cos ^{2} 55^{\circ}
\end{aligned}
$$

and for the $02 \overline{2}$ Bragg reflection peak as

$$
\begin{aligned}
I_{M}(02 \overline{2}) \sim & V_{[\overline{1} 11]} m_{[\overline{1} 11]}^{2}+V_{[\overline{1} 00]} m_{[\overline{1} 00]}^{2} \\
& +V_{[\overline{1} 10]} m_{[\overline{1} 10]}^{2} \cos ^{2} 30^{\circ}+V_{[\overline{1} 0 \overline{1}]} m_{[\overline{1} 1 \overline{1}]}^{2} \cos ^{2} 30^{\circ} \\
& +V_{[010]} m_{[010]}^{2} \cos ^{2} 45^{\circ}+V_{[00 \overline{1}]} m_{[00 \overline{1}]}^{2} \cos ^{2} 45^{\circ} \\
& +V_{[\overline{1} 1 \overline{1}]} m_{[\overline{1} 1 \overline{1}]}^{2} \cos ^{2} 55^{\circ}+V_{[11 \overline{1}]} m_{[11 \overline{1}]}^{2} \cos ^{2} 55^{\circ} .
\end{aligned}
$$

Here and below $V_{[h k l]}$ is the volume of the scattering element magnetized in the direction $[h k l]$.

One can simplify Eqs. (9) and (10) by taking into account first that the [100]- and [110]-type directions are not supported by the form factor of the unit element of the OLS structure. Therefore the model of the unit element magnetization which will be used here for interpretation of experimental data is based on the preferential distribution of magnetization along

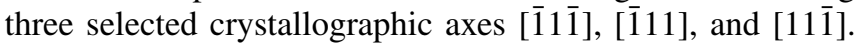
Equations (9) and (10) are rewritten in this case as

$$
\begin{aligned}
I_{M}(20 \overline{2}) \sim & V_{[\overline{1} 1 \overline{1}]} m_{[\overline{1} 1 \overline{1}]}^{2}+V_{[\overline{1} 11]} m_{[\overline{1} 11]}^{2} \cos ^{2} 55^{\circ} \\
& +V_{[11 \overline{1}]} m_{[11 \overline{1}]}^{2} \cos ^{2} 55^{\circ}
\end{aligned}
$$

and

$$
\begin{aligned}
I_{M}(02 \overline{2}) \sim & V_{[\overline{1} 11]} m_{[\overline{1} 11]}^{2}+V_{[\overline{1} 1 \overline{1}]} m_{[\overline{1} 1 \overline{1}]}^{2} \cos ^{2} 55^{\circ} \\
& +V_{[11 \overline{1}]} m_{[11 \overline{1}]}^{2} \cos ^{2} 55^{\circ} .
\end{aligned}
$$

A similar estimation of the interference intensity of the $20 \overline{2}$ and $02 \overline{2}$ reflections leads to the equations

$$
\begin{aligned}
\Delta I(20 \overline{2}) \sim & m_{[\overline{1} 1 \overline{1}]} \cos 19^{\circ}+m_{[\overline{1} 11]} \cos 55^{\circ} \cos 35^{\circ} \\
& +m_{[11 \overline{1}]} \cos ^{2} 55^{\circ} \cos 35^{\circ}
\end{aligned}
$$

and

$$
\begin{aligned}
\Delta I(02 \overline{2}) \sim & V_{[\overline{1} 11]} m_{[\overline{1} 11]} \cos 70^{\circ}+V_{[\overline{1} 1 \overline{1}]} m_{[\overline{1} 1 \overline{1}]} \cos 55^{\circ} \cos 66^{\circ} \\
& +V_{[11 \overline{1}]} m_{[11 \overline{1}]} \cos 55^{\circ} \cos 66^{\circ} .
\end{aligned}
$$

Furthermore, accounting for the fact that the [ $\overline{1} 1 \overline{1}]$ legs are easily magnetized in geometry I and the other two legs along [111] and [11 $\overline{1}]$ are relatively difficult to magnetize, the hysteresis loop of the pure magnetic intensity of the $20 \overline{2}$ reflection [Fig. 11(a)] can be related to the magnetization of the legs parallel to the [ $\overline{1} 1 \overline{1}]$ axis. The small contributions coming from the other legs are noticeable at relatively large fields when the other two legs along [ $\overline{1} 11]$ and [11 $\overline{1}]$ become magnetized. In turn, the hysteresis loop of the magnetic intensity of the $02 \overline{2}$ reflection [Fig. 12(a)] can be related mostly to the magnetization of the legs parallel to the [111] axis. The dominating contribution is imposed on the contribution of the legs parallel to the $[\overline{1} 1 \overline{1}]$ axis. It is especially well seen in the range of small fields, where the legs along the [111] axis are not magnetized but the legs along the [11 $1 \overline{1}]$ axis show a high level of magnetization.

For geometry II, the distribution of magnetization inside the legs is shown in Fig. 14(c). As was considered for geometry I, the legs parallel to [111] are magnetically dead because this is the hard axis for the film and perpendicular to the applied field. In addition, one more type of leg along the [11 1$]$ axis is perpendicular to the field as well, which allows one to consider them also as magnetically dead parts. The other two types of leg along the [ $\overline{1} 11]$ and $[\overline{1} 1 \overline{1}]$ axes are inclined at the angle of $35^{\circ}$ to the field axis. This geometrical disposition leads to the following consequences.

(i) As in geometry I, the OLS film can be divided into individual layers because of the hard [111] axis.

(ii) Each individual layer can be divided into strips along the [110] (along the field) axis because of the second hard [11]1] axis.

(iii) The other two types of leg connection along the [111] and $[\overline{1} 1 \overline{1}]$ axes are rather easily magnetized due to the relatively small angles between the field axis and the leg axes. As a result, one obtains after the simplifications discussed above for the pure magnetic intensity

$$
I_{M}(02 \overline{2}) \sim V_{[\overline{1} 11]} m_{[\overline{1} 11]}^{2}+V_{[\overline{1} 1 \overline{1}]} m_{[\overline{1} 1 \overline{1}]}^{2} \cos ^{2} 55^{\circ}
$$

and for the interference contribution

$\Delta I(02 \overline{2}) \sim V_{[\overline{1} 11]} m_{[\overline{1} 11]} \cos 35^{\circ}+V_{[\overline{1} 1 \overline{1}]} m_{[\overline{1} 1 \overline{1}]} \cos 55^{\circ} \cos 45^{\circ}$.

Thus, the hysteresis loop of the pure magnetic and interference intensities of the $02 \overline{2}$ reflection [Figs. 13(a) and 13(b)] can be related to the magnetization of the legs parallel to the [1111] and $[\overline{1} 1 \overline{1}]$ axes.

The estimation of the magnetic and interference intensities of the $\overline{2} 20$ reflection in geometry II [Fig. 8(b)] shows that it must be zero because the value of the magnetization (perpendicular to $\mathbf{Q}$ and averaged over the unit element) vanishes: $\mathbf{m}_{\perp}=\mathbf{0}$.

\section{Magnetization of the unit element}

The detailed analysis of the magnetization process can also be done on the scale of the single unit element of the OLS shown in Fig. 14(b) for the field applied along the [1 $2 \overline{1}]$ axis (geometry I) and in Fig. 14(c) along the [110] axis (geometry II). For geometry I, the applied field is inclined at $19^{\circ}$ to the [111] axis and its axis passes through the quasitetrahedral plane ( $U M W$ ) via point $M$ to the center of the $(N O P Q)$ quasicubic face or from the center of the quasicubic face $(J K L M)$ via point $O$ in the $(O R T)$ quasitetrahedral plane. For geometry II, the field is applied along the [110] axis and its axis passes through the diagonal of the quasicube face $(M L P Q)$, or $(J K O N)$ and through the quasitetrahedral edge $[U M]$ or $[O T]$, respectively [Fig. 14(c)]. The shaded planes correspond to the $(20 \overline{2})$ and $(02 \overline{2})$ reflection planes [Fig. 14(b)] and to the $(02 \overline{2})$ reflection plane [Fig. 14(c)], which are visible in the corresponding geometries of scattering. For 
magnetic diffraction the orientations of the magnetization with respect to the reflecting crystallographic planes are important. The projection of the magnetization onto these planes, $\mathbf{m}_{\perp Q}$, determines the intensity of the magnetic neutron scattering.

Again, to understand the distribution of the magnetization density and its orientations throughout the unit element, one should realize that the vertices with the [111] axes perpendicular to the field are not magnetized due to the shape anisotropy of the film. For geometry I, these vertices are those at the point $K$ and the point $Q$ for the quasicube, and those at point $V$ and point $S$ for the quasitetrahedra. These leg connections can be considered as magnetically dead elements. For geometry II, there are more such vertices with axes perpendicular to the field axis, which can be considered as magnetically dead elements. [Fig. 14(c)]. These are the vertices at points $K$, $Q, L$, and $N$ for the quasicube and those at points $V, S, W$, and $R$ for the quasitetrahedra. In fact, some of the vertices belonging to the quasicube are parts of the same legs that connect it to the quasitetrahedra. Therefore, some of the point vertices are equivalent to other points within the same unit element. These equivalent points are $S$ and $Q, K$ and $V, T$ and $J, R$ and $L, W$ and $N$, and $U$ and $P$. Thus, the value and the orientation of the magnetization at one point must be exactly the same as at the other.

The magnetic field applied in geometry I can easily magnetize the vertices at the points $M$ and $O$ because the axis of these leg connection between quasicube and quasitetrahedra is only $19^{\circ}$ inclined to the field axis [1릴. The other leg connections at points $U$ and $V$ (and their equivalents $P$ and $K$ ) as well as at points $R$ and $T$ (and their equivalents $L$ and $J$ ) are more difficult to magnetize because their axes are $66^{\circ}$ inclined to the field axis. The magnetization vector will strongly deviate from the applied field axis in these parts of the unit element. The direction is determined by the anisotropy of the inverse OLS and coincides with the [111]-type axes.

The magnetic field applied in geometry II can equally magnetize the vertices at the points $T, O, J$ and $U, M, P$ because the axis of these leg connection is only $35^{\circ}$ inclined to the field axis [1ㅣㅣㅇ. The other leg connections cannot be magnetized because their axes are $90^{\circ}$ inclined to the field axis. Thus the magnetization tends to orient along the allowed [111]-type axes in the intermediate field range as shown in Fig. 14. The forbidden (perpendicular to the field or to the film plane) [111]-type axes are considered to be magnetically dead. These magnetically dead connections produce the biggest contribution to the diffuse scattering measured as $A_{D}$.

The magnetization thus modeled within the single unit element should be projected onto the corresponding reflecting planes to calculate the scattering intensity for the pure magnetic contribution and for the interference contribution. Thus, one comes to the expressions given above.

\section{CONCLUDING REMARKS}

(1) Ultrasmall-angle diffraction of the synchrotron radiation is shown to be a much more effective tool for studying the structure of opal-like structures due to its high brilliance and high resolution. Polarized neutron diffraction remains irreplaceable in the study of magnetic structures.

(2) The ultrasmall-angle diffraction of the synchrotron radiation allowed us to establish the crystallographic structure of the inverse opal-like structure, which is found to be close to face-centered cubic with the lattice constant of $640 \mathrm{~nm}$.

(3) The polarized small-angle neutron diffraction demonstrated that the 3D geometrical shape of the structure leads to a complex distribution of the magnetization in the sample, which is far from being simply uniform. We determined the profile of the magnetization within the net structure of the OLS.

(4) The sample of OLS demonstrates clearly the easy-plane (111) anisotropy caused by the fact that the sample is a film. The inverse Co OLS dictates the structural anisotropy, as well, and in the magnetization process with an in-plane applied field we should consider three easy magnetization axes only: [11 $\overline{1}],[\overline{1} 11]$, and [ $\overline{1} 1 \overline{1}]$. The sample, therefore, is divided into a number of individual magnetic layers in the [111] direction, which are magnetized independently of each other. In geometry II, additionally, each individual layer is divided into strips along the [110] axis (which is the same as the field

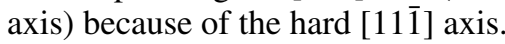

\section{ACKNOWLEDGMENTS}

The PNPI and SPSU teams acknowledge HelmholtzZentrum Geesthacht for hospitality and financial support. The work is supported in part by the RFBR (Project No 10-0200634), the Program of the Russian Government Development of the Scientific Potential of the High School (Grants No. 2.1.1/4661 and No. 2.2.2.3/8155), and the RF Program (Grant No. 02.740.11.5186). We give special thanks to D. Menzel and J. Schoenes from TU Braunschweig for the opportunity to conduct SQUID measurements in the frame of the DAAD programs Mikhail Lomonosov and Leonhard Euler. The Interdisciplinary Resource Center of Nanotechnology of St. Petersburg State University is acknowledged for the SEM measurements. We thank K. Kvashnina and D. Detollenaere from the DUBBLE beamline for their excellent support. The authors acknowledge Netherlands Organization for Scientific Research (NWO) for financial support.
${ }^{1}$ Y. Xia, Adv. Mater. 13, 369 (2001).

${ }^{2}$ S. A. Jonson, P. J. Ollivier, and T. E. Mallouk, Science 283, 963 (1999).

${ }^{3}$ P. Jiang, K. S. Hwang, D. M. Mittleman, J. E. Bertone, and V. L. Colvin, J. Am. Chem. Soc. 121, 11630 (1999).

${ }^{4}$ T. Simuda, Y. Wada, T. Kitamura, and S. Yanagida, Chem. Commun. 17, 1613 (2000)
${ }^{5}$ K. Busch and S. John, Phys. Rev. Lett. 83, 967 (1999).

${ }^{6}$ E. Yablonovitch, Nature (London) 401, 539 (1999).

${ }^{7}$ O. K. Alimov, T. T. Basiev, Yu. V. Orlovskii, V. V. Osiko, and M. I. Samoilovich, Quantum Electron. 38, 665 (2008).

${ }^{8}$ B. T. Holland, C. F. Blanford, and A. Stein, Science 281, 538 (1998).

${ }^{9}$ J. E. G. J. Wijnhoven and W. L. Vos, Science 281, 802 (1998). 
${ }^{10}$ H. Yan, C. F. Blanford, B. T. Holland, W. H. Smyrl, and A. Stein, Chem. Mater. 12, 1134 (2000).

${ }^{11}$ F. Chen, C. Xia, and M. Liu, Chem. Lett. 30, 1032 (2001).

${ }^{12}$ A. A. Zakhidov, R. H. Baughman, Z. Iqbal, C. Cui, I. Khayrullin, S. O. Dantas, J. Marti, and V. G. Ralchenko, Science 282, 897 (1998).

${ }^{13}$ Y. A. Vlasov, N. Yao, and D. J. Norris, Adv. Mater. 11, 165 (1999).

${ }^{14}$ P. V. Braun and P. Wiltzius, Nature (London) 402, 603 (1999).

${ }^{15}$ A. Blanco, E. Chomski, S. Grabtchak, M. Ibisate, S. John, S. W. Leonard, C. Lopez, F. Meseguer, H. Miguez, J. P. Mondia, G. A. Ozin, O. Toader, and H. M. van Driel, Nature (London) 405, 437 (2000).

${ }^{16}$ Z. Lei, J. Li, Y. Ke, Y. Zhang, H. Wang, and G. He, J. Mater. Chem. 11, 1778 (2001)

${ }^{17}$ A. Lungu, M. Bleiweiss, J. Amirzadeh, S. Saygi, A. Dimofte, M. Yin, Z. Iqbal, and T. Datta, Physica C 349, 1 (2001).

${ }^{18}$ A. A. Zhukov, E. T. Filby, M. A. Ghanem, P. N. Barlett, and P. A. J. de Groot, Physica C 404, 455 (2004).

${ }^{19}$ A. E. Aliev, S. B. Lee, A. A. Zakhidov, and R. H. Baughman, Physica C 453, 15 (2007).

${ }^{20}$ O. D. Velev, P. M. Tessier, A. M. Lenhoff, and E. W. Kaner, Nature (London) 401, 548 (1999).

${ }^{21}$ P. Jiang, J. Cizeron, J. F. Bertone, and V. L. Colvin, J. Am. Chem. Soc. 121, 7957 (1999).

${ }^{22}$ H. Yan, C. F. Blanford, B. T. Holland, M. Parent, W. H. Smyrl, and A. Stein, Adv. Mater. 11, 1003 (1999).

${ }^{23}$ L. Xu, W. L. Zhou, C. Fromer, R. H. Baughman, A. A. Zakhidov, L. Malkinski, J.-Q. Wang, and J. B. Wiley, Chem. Commun. 17, 997 (2000).

${ }^{24}$ J. E. G. J. Wijnhoven, S. J. M. Zevenhuizen, M. A. Hendriks, D. Vanmaekelbergh, J. J. Kelly, and W. L. Vos, Adv. Mater. 12, 888 (2000).

${ }^{25}$ P. N. Bartlett, P. R. Birkin, and M. A. Ghanem, Chem. Commun. 17, 1671 (2000).

${ }^{26}$ L. Xu, L. D. Tung, L. Spinu, A. A. Zakhidov, R. H. Baughman, and J. B. Wiley, Adv. Mater. 15, 1562 (2003).
${ }^{27}$ J. G. Fleming, S. Y. Lin I. El-Kady, R. Biswas, and K. M. Ho, Nature (London) 417, 52 (2002).

${ }^{28}$ S. Y. Lin, J. Moreno, and J. G. Fleming, Appl. Phys. Lett. 83, 380 (2003).

${ }^{29}$ D. C. Look, Mater. Sci. Eng. B 80, 383 (2001).

${ }^{30}$ C. Baratto, G. Faglia, G. Sberveglieri, A. Sutti, G. Calestani, and C. Dionigi, IEEE Sensors 1196 (2005).

${ }^{31}$ G. Herzer, J. Magn. Magn. Mater. 112, 258 (1992).

${ }^{32}$ W. Wernsdorfer, E.B. Orozco, K. Hasselbach, A. Benoit, B. Barbara, N. Demoncy, A. Loiseau, H. Pascard, and D. Mailly, Phys. Rev. Lett. 78, 1791 (1997).

${ }^{33}$ J. F. Loffler, H.-B. Braun, and W. Wagner, Phys. Rev. Lett. 85, 1990 (2000).

${ }^{34}$ J. F. Loffler, H.-B. Braun, W. Wagner, G. Kostorz, and A. Wiedenmann, Phys. Rev. B 71, 134410 (2005).

${ }^{35}$ S. V. Grigoriev, K. S. Napolskii, N. A. Grigoryeva, A. V. Vasilieva, A. A. Mistonov, D. Yu. Chernyshov, A. V. Petukhov, D. V. Belov, A. A. Eliseev, A. V. Lukashin, Yu. D. Tretyakov, A. S. Sinitskii, and H. Eckerlebe, Phys. Rev. B 79, 045123 (2009).

${ }^{36}$ A. I. Plekhanov, D. V. Kalinin, and V. V. Serdobintseva, Ross. Nanotekh. 1, 245 (2006) (in Russian).

${ }^{37}$ A. V. Petukhov, J. H. J. Thijssen, D. C. 't Hart, A. Imhof, A. van Blaaderen, I. P. Dolbnya, A. Snigirev, A. Moussad, and I. Snigireva, J. Appl. Crystallogr. 39, 1822 (2006).

${ }^{38}$ J. H. J. Thijssen, A. V. Petukhov, D. C. 't Hart, A. Imhof, K. van der Werf, R. E. I. Schropp, and A. van Blaaderen, Adv. Mater. 18, 1662 (2006).

${ }^{39}$ V. V. Abramova, A. S. Sinitskii, N. A. Grigor'eva, S. V. Grigor'ev, D. V. Belov, A. V. Petukhov, A. A. Mistonov, A. V. Vasil'eva, and Yu. D. Tret'yakov, JETP 109, 29 (2009).

${ }^{40}$ J. Hilhorst, V. V. Abramova, A. Sinitskii, N. A. Sapoletova, K. S. Napolskii, A. A. Eliseev, D. V. Byelov, N. A. Grigoryeva, A. V. Vasilieva, W. G. Bouwman, K. Kvashnina, A. Snigirev, S. V. Grigoriev, and A. V. Petukhov, Langmuir 25, 10408 (2009).

${ }^{41}$ G. E. Bacon, Neutron Diffraction (Clarendon Press, Oxford, 1955).

${ }^{42}$ L. A. Feigin and D. I. Svergun, Structure Analysis by Small-Angle $X$-Ray and Neutron Scattering (Plenum Press, New York, 1987). 